\title{
Anti-Stress and Glial Differentiation Effects of a Novel Combination of Cucurbitacin B and Withanone (CucWi-N): Experimental Evidence
}

\author{
Sukant Garg ${ }^{a, b}$ Sunil C. Kaul ${ }^{a}$ Renu Wadhwa ${ }^{a, b}$ \\ a DBT-AIST International laboratory for Advanced Biomedicine (DAILAB), National Institute of Advanced \\ Industrial Science and Technology (AIST), Tsukuba, Japan; ${ }^{\mathrm{b}}$ School of Integrative and Global Majors, \\ University of Tsukuba, Tsukuba, Japan
}

\section{Keywords}

Cucurbitacin B · Withanone - Glial differentiation ·

Stress · Therapeutics

\begin{abstract}
Background: Natural extracts and compounds used in traditional home medicine are known for their safety and a variety of health promoting and therapeutic potentials. In contrast to the single molecule mediated targets, the combinational therapies are preferred for their multi-functional and limited toxic regimens and may be useful for disease therapeutics as well as to increase the quality of life during a variety of environmental stresses. Purpose: We aimed to combine the active ingredients of Chinese (Helicteres angustifolia) and Indian (Withania somnifera) ginsengs to develop a natural, efficient, and welfare combinatorial mixture with high anti-stress and glial differentiation potentials. Methods: Using cultured cells as a model system, we developed a combination of active ingredients of Chinese (Cucurbitacin
\end{abstract}

B [Cuc]) and Indian (Withanone [Wi-N]) ginsengs. Eleven chemical models of environmental stresses were used. Cytotoxicity studies were performed using human skin fibroblast for anti-stress and rat glioma cells for glial differentiation effects. Results: We demonstrate that the novel combination of Cuc and Wi-N, CucWi-N, was non-toxic to normal cells. It caused stress protection in assays using normal human fibroblasts subjected to a variety of stresses. Of note, cells showed remarkable protection against oxidative and UV stresses and marked by decrease in DNA damage and reactive oxygen species. We examined and found the glial differentiation potential of CucWi-N in rat glioblastoma cells. CucWi-N clearly induced differentiation phenotype, well-marked with upregulation of GAP43, MAP2, and GFAP, which have been shown to play a key role in glial differentiation. Conclusion: These data demonstrate anti-stress and glial differentiation potential of CucWi-N (a novel combination of Cuc and Wi-N) that could be recruited in nutraceutical and pharmaceutical avenues and hence warrant further evaluation and mechanistic studies.

\section{KARGER}

(c) 2018 S. Karger AG, Basel

E-Mail karger@karger.com

www.karger.com/aon
Renu Wadhwa

National Institute of Advanced Industrial Science \& Technology (AIST)

Central 5-41, Tsukuba 3058565 (Japan)

E-Mail renu-wadhwa@ aist.go.jp 


\section{Introduction}

Stress is a state of living system, wherein an absence of essential or presence of an undesired agent triggers molecular and functional changes in survival signaling, leading to reversible and/or permanent changes. It has been implicated in a large variety of diseases, most of these emerge from decline in tissue functions, which is also a hall-mark of aging $[1,2]$. In view of this, stress has been entitled as an important aging-instigating and aggravating factor; anti-stress reagents are predicted to delay agerelated tissue malfunctions and increase quality of life (QOL) especially in the later years of life [3]. Cancer has also been characterized as a stressed state of cells wherein cells lose their normal functions, and dedifferentiate and proliferate infinitely disrupting normal body functions $[4,5]$. Cancer incidence has been seen to increase exponentially due to prolonged human lifespan in last 3-4 decades amounting to old-age societies world-wide, and increase in environmental stresses due to industrialization and heavy use of chemicals in daily life [6]. At the same time, neurodegenerative diseases that mark old age afflict QOL at individual, family, social levels at large, and become high burden on social and health-care systems. In view of these, research on the identification of anti-stress natural reagents, studying their molecular mechanisms of action and use in preventive and therapeutic use have been prioritized.

Cell culture has been established as a reliable model system for not only in stress biology research but also for screening natural and synthetic anti-stress compounds [7]. Amongst a large variety of chemical models of stress, such as benzo[a]pyrene for smoke, sodium arsenite for heavy metal, 2-nitro-9-fluorenone for diesel, bisphenol A bis(chloroformate) for plastics, cobalt chloride for hypoxia, hydrogen peroxide for oxidative stress, and UV radiations for DNA damage stress, the last two have been used most widely in the laboratory using cultured mammalian cells [8]. A large number of natural and synthetic anti-oxidant compounds that cause either attenuation of production or destruction of reactive oxygen species (ROS) (charged super-ions continually generated and released in the cells) have been identified and promoted to nutraceutical as well as pharmaceutical industries [9-11]. Molecular mechanisms of their action and specific effects either on cancer or age-related neurodegenerative diseases are only beginning to be attempted and resolved [12, 13].

Screening of anticancer compounds is relatively easy as it relies on identification of cytotoxic properties and cell death mechanisms. The latter could be achieved in shortterm experiments. On the other hand, screening of compounds with potentials to modulate stress and brain functions is challenging as it demands identification of nontoxic and safe compounds, and characterization of functional properties of living cells in long-term assays. Prevention and control of stress-related anomalies is a complex and complicated task as it requires comprehensive characterization of chemical as well as physiological effects on normal as well as diseased cell and tissue states. Whereas single synthetic molecules were favored for targeted therapies in the past, they have been associated with shortcomings including toxicity, high cost, and drug resistance phenotypes $[14,15]$. Natural home medicine ingredients have been shown to provide a safer and holistic remedies for treatment of stress and cancer and are often predicted to offer additional benefits such as higher and broad spectrum efficacy $[16,17]$.

We have earlier demonstrated that ashwagandha (Indian ginseng)-derived withanolides possess significant anticancer activity in in vitro and in vivo [18-25]. Low doses of the bioactive withanolides (Withaferin A [Wi-A] and Withanone [Wi-N]) caused protection of normal cells against oxidative and DNA damage [26, 27]. Molecular studies have demonstrated activation of tumor suppressor function, oxidative and DNA damage selectively in cancer cells [21, 24]. Furthermore, methoxy-Withaferin A, although inactive for cancer cells, was shown to possess anti-stress potential in normal cells [28] suggesting side benefits of holistic extracts used in traditional home medicine that has been in practice for centuries in Ayurveda. On similar lines, we earlier identified anticancer activity in the root extract of Chinese ginseng, Helicteres angustifolia [29]. However, the active component of the extract, Cucurbitacin B (Cuc) showed cytotoxicity not only on cancer but also on normal cells [30]. In view of these findings, we engineered a combination of $\mathrm{Cuc}$ and $\mathrm{Wi}-\mathrm{N}$ (CucWi-N) that was selectively toxic to cancer cells (unpublished data). In the present study, we characterized the effect of CucWi-N on normal cells under a variety of stressed conditions. We found that CucWi-N not only protects normal cells against a variety of stresses, but also triggers differentiation in glioblastoma cells suggesting its potential in enhancing QOL and brain functions.

\section{Materials and Methods}

Cells and Reagents

Human normal skin fibroblasts (TIG-3) and rat glioblastoma cells (C6) were cultured in Dulbecco's modified Eagle's medium 
(Invitrogen) supplemented with 5\% fetal bovine serum and 1\% penicillin/streptomycin in a humidified incubator $\left(37^{\circ} \mathrm{C}\right.$ and $5 \%$ $\mathrm{CO}_{2}$ ). Cuc (Sigma-Aldrich) and Wi-N (Funakoshi) were dissolved in dimethyl sulfoxide (DMSO) to prepare stock and working solutions. Antibodies against GAP43 (SC-33705, Santa Cruz), MAP2 (M3696, Sigma Aldrich), GFAP (G9269, Sigma Aldrich), mortalin [31], and $\beta$-actin (ab49900, AbCam) proteins were used for immunoblotting and immunostaining.

\section{Cell Viability, Stress, and Anti-Stress Assays}

The cytotoxicities of $\mathrm{Cuc}, \mathrm{Wi}-\mathrm{N}$, and $\mathrm{CucWi}-\mathrm{N}$ was determined by 3-(4,5-dimethylthiazol-2-yl)-2,5-diphenyltetrazolium bromide (MTT)-based assay. Cells $\left(2 \times 10^{3} /\right.$ well $)$ were plated in 96-well plate, allowed to settle overnight, and treated with varying doses of the compounds. The control (DMSO) or treated cells were incubated for $48 \mathrm{~h}$ followed by addition of $10 \mu \mathrm{L}$ of phosphate buffered saline (PBS) containing $5 \mathrm{mg} / \mathrm{mL}$ MTT (M6494, Life Technologies), and further incubated for $4 \mathrm{~h}$. Media containing MTT was aspirated and replaced with DMSO. The plates were placed on a shaker for 5 min followed by measurement of optical density at $570 \mathrm{~nm}$ using Tecan infinite M200 ${ }^{\circledR}$ Pro microplate reader (Tecan Group Ltd., Mannedorf, Switzerland). Cell viability was calculated in percentage against the control. Cells were stressed with indicated chemical models of stress for $60 \mathrm{~min}$, followed by recovery in either control or CucWi-N-supplemented medium for $48 \mathrm{~h}$. UV chamber (FS-800, FUNA ${ }^{\circledR}$-UV-linker) was used to induce UVradiation stress. For visual observations, cells were fixed in $100 \%$ methanol, incubated with $0.5 \%$ crystal violet stain for $2 \mathrm{~h}$, excessively washed in PBS, air dried and visualized under the microscope.

\section{DNA Damage and ROS Detection}

In order to explore DNA damaging effect of CucWi-N, comet assay was done using single cell gel neutral comet assay (Trevigen's Comet Assay ${ }^{\circledR}$ ) electrophoresis system following the manufacturer's protocol. ROS levels were determined by ROS Live Stain Assay performed using commercially available Image-IT ${ }^{\mathrm{TM}}$ LIVE Green Reactive Oxygen Species Detection Kit, for microscopy (I36007, Invitrogen) following the manufacturer's protocol.

\section{Immunoblotting}

Control and treated cells were harvested and washed with PBS (X2), followed by lysis in RIPA buffer (89900, Thermo Fisher) containing complete protease inhibitor cocktail (4693159001, Roche Applied Science) on ice for $45 \mathrm{~min}$. Lysates were separated on a SDS-polyacrylamide gel using Mini-Protean ${ }^{\circledR}$ Tetra cell equipment (Bio-Rad, Hercules, CA, USA), and subjected to Western blotting [24] using protein-specific antibodies as indicated and horseradish peroxidase-conjugated secondary HRP antibody (31430, Thermo Fisher or 31460, Thermo Fisher). Blots were developed using chemiluminescence solution (GE Healthcare, UK) and visualized using Lumino Image Analyzer (LAS3000-mini; Fuji Film).

\section{Immunostaining}

Cells were plated on coverslips placed in 12-well plates and allowed to settle overnight, followed by culture either in control or CucWi-N-supplemented medium. Control and treated cells were fixed in methanol: acetone (1:1) on ice for $5 \mathrm{~min}$, rinsed with PBS (X3), permeabilized with $0.2 \%$ PBST $(0.2 \%$ TritonX-100 in
PBS) for $20 \mathrm{~min}$, washed with PBS for $15 \mathrm{~min}$, and blocked with $2 \%$ bovine serum albumin protein dissolved in PBST for $45 \mathrm{~min}$. Fixed cells were immunostained using primary antibodies (as indicated) and matched secondary antibodies as described earlier [24].

\section{Statistical Analysis}

All the calculations were done using Microsoft ${ }^{\circledR}$ office. Statistical significance was calculated by unpaired t test of GraphPad software $^{\circledR}$ (2017) using mean, SD, and N from 3 independent experiments, and shown as ${ }^{*} p<0.05,{ }^{* *} p<0.01,{ }^{* * *} p<0.001$.

\section{Results}

\section{CucWi-N Possesses Anti-Stress Potential}

In order to investigate the anti-stress potential of CucWi-N, dose response of human normal skin fibroblasts to CucWi-N was first determined. As shown in Figure 1a, whereas Cuc 5-10 $\mathrm{MM}$ begins to show toxicity to TIG-3 cells, its combination with Wi-N (2.5$5.0 \mu \mathrm{M})$ was inert. We then established the response of cells to the treatment with $\mathrm{H}_{2} \mathrm{O}_{2}$ and UV. Dose-dependent decrease in viability was obtained in cells treated with $\mathrm{H}_{2} \mathrm{O}_{2}$ (Fig. 1b) as well as UV (Fig. 1c). By multiple assays, we chose $650 \mu \mathrm{M}$ of $\mathrm{H}_{2} \mathrm{O}_{2}$ and $\mathrm{UV} 5 \mathrm{~mJ} / \mathrm{cm}^{2}$ $\left(\mathrm{IC}_{50}\right)$ as appropriate stress inducing conditions. We then used 9 other chemical models of various environmental stresses (Fig. 1d) and determined their effect on TIG-3 cell viability. By multiple experiments, $\mathrm{IC}_{10^{-}}$ $\mathrm{IC}_{50}$ doses of stresses were determined previously [8]; $\sim \mathrm{IC}_{20}$ doses were used to determine the anti-stress effect of CucWi-N. By several independent experiments, we found that CucWi-N caused increase in survival of cells exposed to chemical models of stresses including diesel (2-nitro-9-fluorenone), anger (epinephrine), cans (vanadium oxide), plastics (bisphenol A bis(chloroformate), battery (cadmium nitrate tetrahydrate), hypoxia (cobalt chloride), paint (formaldehyde), oxidation (hydrogen peroxide), and DNA damage (ultraviolet radiations; Fig. $1 \mathrm{~d}-\mathrm{f}$ ). We, next, chose UV and $\mathrm{H}_{2} \mathrm{O}_{2}$ stresses and determined their effect on control and CucWi-N-treated cells. As shown in Figure 2a, Comet assay showed increase in tail DNA (indicative of DNA damage) in UV-treated cells. Of note, CucWi-Ntreated control cells did not show any increase in tail DNA (Fig. 2a). ROS assay in control and $\mathrm{H}_{2} \mathrm{O}_{2}$-stressed cells with/without CucWi-N clearly revealed its increase in $\mathrm{H}_{2} \mathrm{O}_{2}$-treated cells and its decrease when cells were recovered in CucWi-N-supplemented medium (Fig. 2b). 


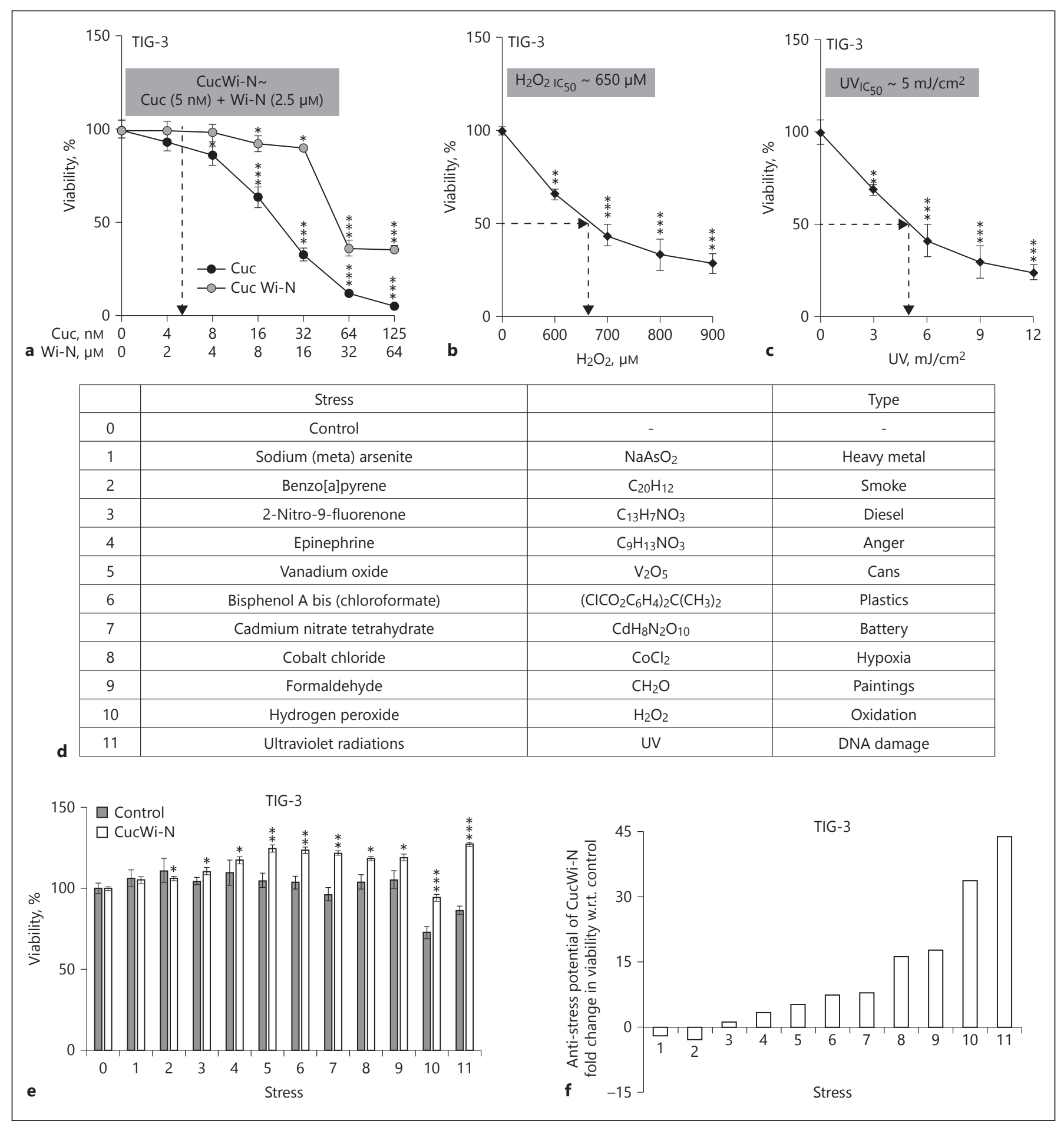

Fig. 1. Anti-stress potential of $\mathrm{CucWi}-\mathrm{N}$ in normal skin fibroblasts (TIG-3). a Dose response of cells to Cuc and CucWi-N during $48 \mathrm{~h}$ treatment; (b), dose titration and determination of IC50 of $48 \mathrm{~h} \mathrm{H} 2 \mathrm{O} 2$ treatment; (c), dose titration and determination of IC50 of 48 h UV treatment; (d), tabulated chemical/physical models of environmental stresses; (e), viability of control and CucWi-
$\mathrm{N}$-treated cells exposed to stresses as indicated by numbered with reference to d; (f), graphical representation of the percent fold change in cell viability of cells exposed to stresses and recovery in CucWi-N supplemented medium with respect to the ones recovered in normal medium. CucWi-N, Cucurbitacin B and Withanone. ${ }^{*} p<0.05,{ }^{* *} p<0.01,{ }^{* * *} p<0.001$. 


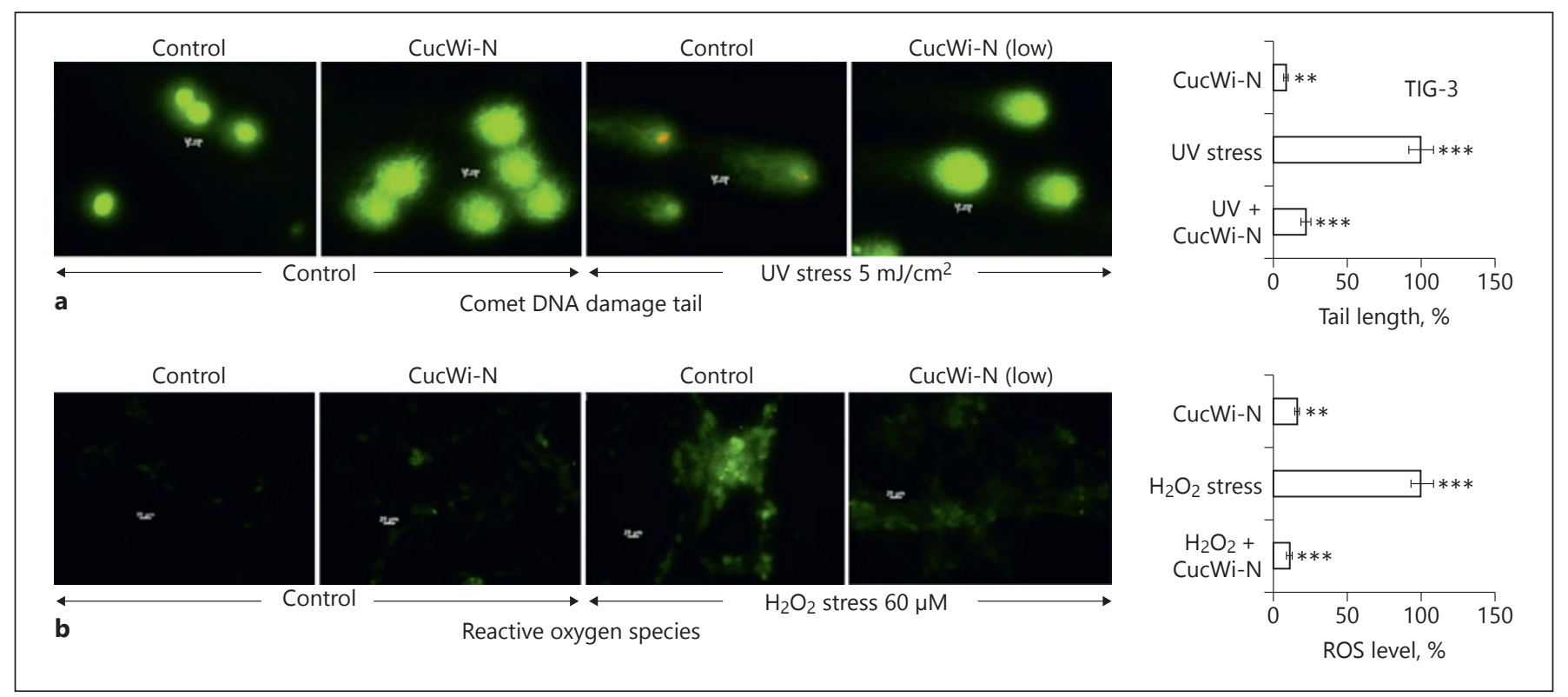

Fig. 2. Effect of CucWi-N on stress-induced increase in DNA damage and ROS levels in normal skin fibroblasts (TIG-3). a Comet assay images of control and CucWi-N-treated TIG-3 cells with/ without UV stress $(5 \mathrm{~mJ} / \mathrm{cm} 2)$. Length of the "tail" corresponds to the extent of DNA damage. Whereas UV-treated cells showed increase in tail DNA, CucWi-N treatment caused decrease in tail length; (b), ROS assay images of control and $\mathrm{H} 2 \mathrm{O} 2(600 \mu \mathrm{M})$ stressed cells recovered either in control or CucWi-N-supplemented medium. CucWi-N-treated cells showed protection against $\mathrm{H} 2 \mathrm{O} 2$-induced increase in ROS. Quantitation of (a) and (b) is shown on the right panels, respectively. ${ }^{*} p<0.05,{ }^{* *} p<0.01$, *** $p<0.001$.

\section{CucWi-N has Glial Differentiation Potential}

Based on the anti-stress activity of CucWi-N as described above, we next questioned whether it has an ability to promote brain function and improve QOL by enhancing the differentiation capacity of brain derived cells. As a model, we used rat glioma (C6) cells that offer an easy culture system for assessment of glial differentiation potential of drugs [22]. Dose response of C6 cells to CucWi-N was first established (Fig. 3a) and 3 combinatorial doses were selected for analysis of their glial differentiation potential. Cells were subjected to low $\left(\mathrm{IC}_{01}\right)$, medium $\left(\mathrm{IC}_{10}\right)$ and high $\left(\mathrm{IC}_{50}\right)$ doses of $\mathrm{CucWi}-\mathrm{N}$ and first examined for differentiation morphology during 14 days of culture. As shown in Figure 3b, cells showed distinct differentiation phenotype in a dose-dependent manner. Of note, the high dose showed toxicity and differentiation together suggesting that it may be recruited as a drug for differentiation therapy for glioblastoma tumors that are extremely difficult to be treated by radio and chemotherapy regimens. Differentiation therapy may offer a milder, safer, and functional alternative in such cases. In order to confirm the differentiation phenotype, we generated GFP-tagged cells and confirmed their CucWi-N-induced differentiation in a dose-depen- dent manner (Fig. 3b). Furthermore, we examined the expression level of proteins that play a key role in glial differentiation. As shown in Figure 4a, cells treated with CucWi-N showed upregulation in GAP43, MAP2, and GFAP proteins in a dose-dependent manner. The findings were endorsed by Western blot analysis using specific antibodies (Fig. 4b).

\section{Discussion}

Stress is a state of a living system, biologically perceived as a disturbance in the homeostasis [2]. It involves cascade of responses including structural and functional changes in biomolecules yielding peculiar disease phenotypes that may range from common cold, inflammation, decline in brain functions, and cancer. Although connected to almost all known diseases, stress is not defined as a disease by itself and is an unavoidable part of normal life. Traditional home medicine systems offer a huge and healthy resource of information on herbs, their extracts, and pure compounds with anti-stress properties that could be recruited for enhancing QOL in the normal or diseased states. Whereas pure compounds are preferred 


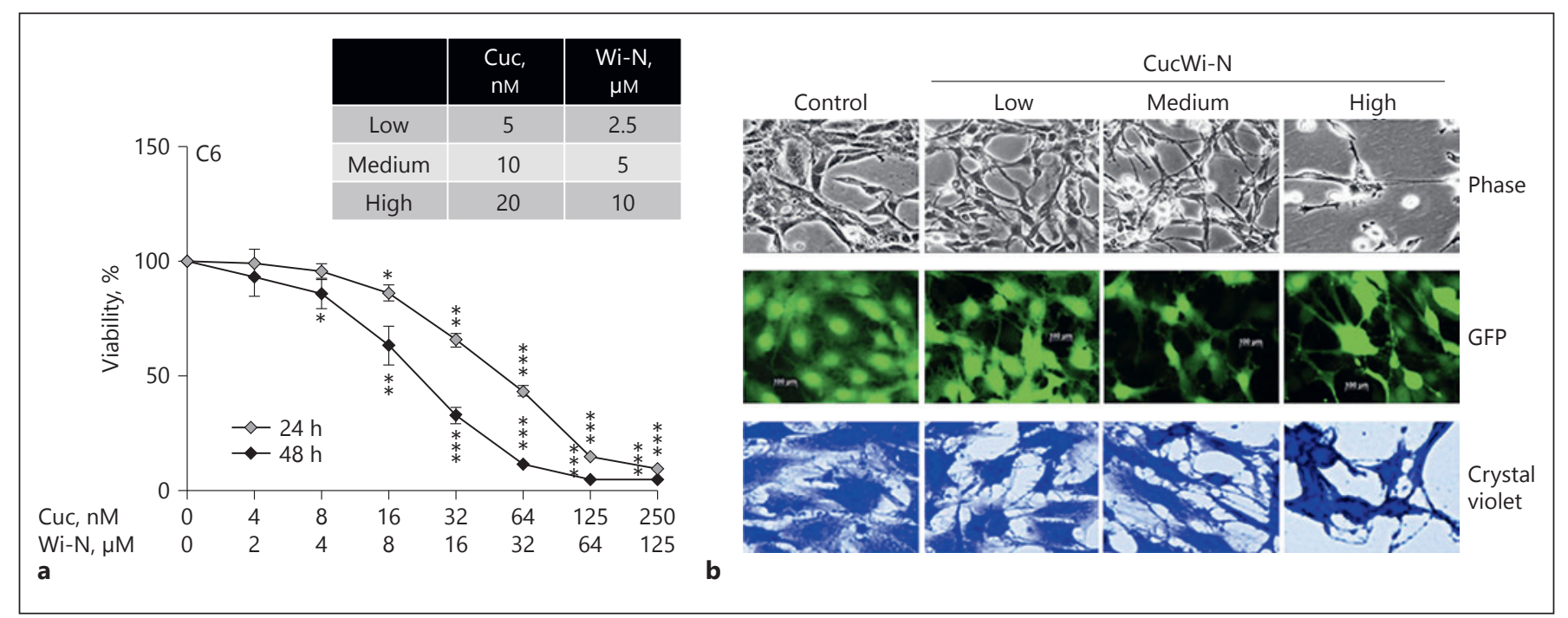

Fig. 3. Effect of CucWi-N on viability and differentiation phenotypes of rat glial fibroblasts (C6 cells). a Cytotoxicity and dose response of C6 cells to CucWi-N: IC01 (low), IC10 (medium) and IC50 (high) were determined for 24 and $48 \mathrm{~h}$ treatment regimens;

for botanical drug development, extracts are preferred for disease preventive and boosting QOL.

Brain function is an extremely important aspect of stress biology. It is directly involved in initiation of stress signaling, persistence, and attenuation. Although several synthetic and natural anti-stress molecules are available as nutraceuticals in the market, they are limited in terms of experimental evidence to their claimed effects and often raise undesired adverse effects [32]. There is a compelling need to develop natural, efficient, and welfare combinatorial compounds/extracts with broad and safe spectrum of effects in functional preventive and therapeutic medicine. Glial cells (astrocytes, microglia, oligodendrocytes, Schwann cells, and radial glia) are the major constituent of the central nervous system that function to provide anchorage, nutrients, oxygen, and insulation to the neurons and excrete waste away $[33,34]$. They are an extremely important constituent of memory, motor, and other functions of CNS, and are subjected to high internal and external stresses. As a result, the incidence of a variety of diseases including cancer in glial cells (glioblastoma) is high. Such diseases are further complicated by extremely poor prognosis and difficult to treat. Brain functions in normal and diseased states are highly influenced by stress and hence demand interventions with natural, efficient, and welfare compounds.

Several studies have earlier reported therapeutic potential of Cuc for treatment of cancer, cardiac, liver, lung, (b), Morphology of cells treated with indicated doses of CucWi-N. Phase contrast, GFP, and crystal violet-stained images are shown. ${ }^{*} p<0.05,{ }^{* *} p<0.01,{ }^{* * *} p<0.001$.

neuronal and skin injuries, systemic infections, generalized inflammation, sex-related disorders, and adipocyte differentiation abnormalities [30]. It has been shown to possess anti-oxidant, anti-viral, anti-pyretic, analgesic, and anti-malarial properties. Cuc protected the heart against pressure overload cardiac hypertrophy and fibrosis, prevented sepsis-induced acute lung injury, counteracted psoriatic lesions and neuronal injuries, and fatty liver in various in vivo and clinical systems. It was shown to inhibit growth of human glioblastoma multiforme cells by intense disturbance in its internal cytoskeleton [35]. Within 15-30 min of treatment, it caused loss of pseudopodia, mutlinucleation, and skirting up of cells associated with disruption of actin and microtubules that was immediately followed by the activation of cell survival pathways. Touihri-Barakati et al. [36] also suggested the role of integrin in Cuc-mediated inhibition of proliferation and glio-differentiation. Its therapeutic value has been dampened by severe toxicity in vivo [37] as shown by symptoms including diarrheal gastric upset, syncope, and respiratory failure [30]. We also previously reported its toxicity on cultured human normal fibroblasts [29].

$\mathrm{Wi}-\mathrm{N}\left(\mathrm{C}_{28} \mathrm{H}_{38} \mathrm{O}_{6}\right)$, on the other hand, is one of the relatively less studied bioactive that is derived from Withania somnifera. It was shown to cause selective cytotoxicity on cancer cells by irreversibly damaging DNA repair mechanisms, disturbing the cellular assembly and organization, and mortalin-p53 function $[18,19,24]$. 


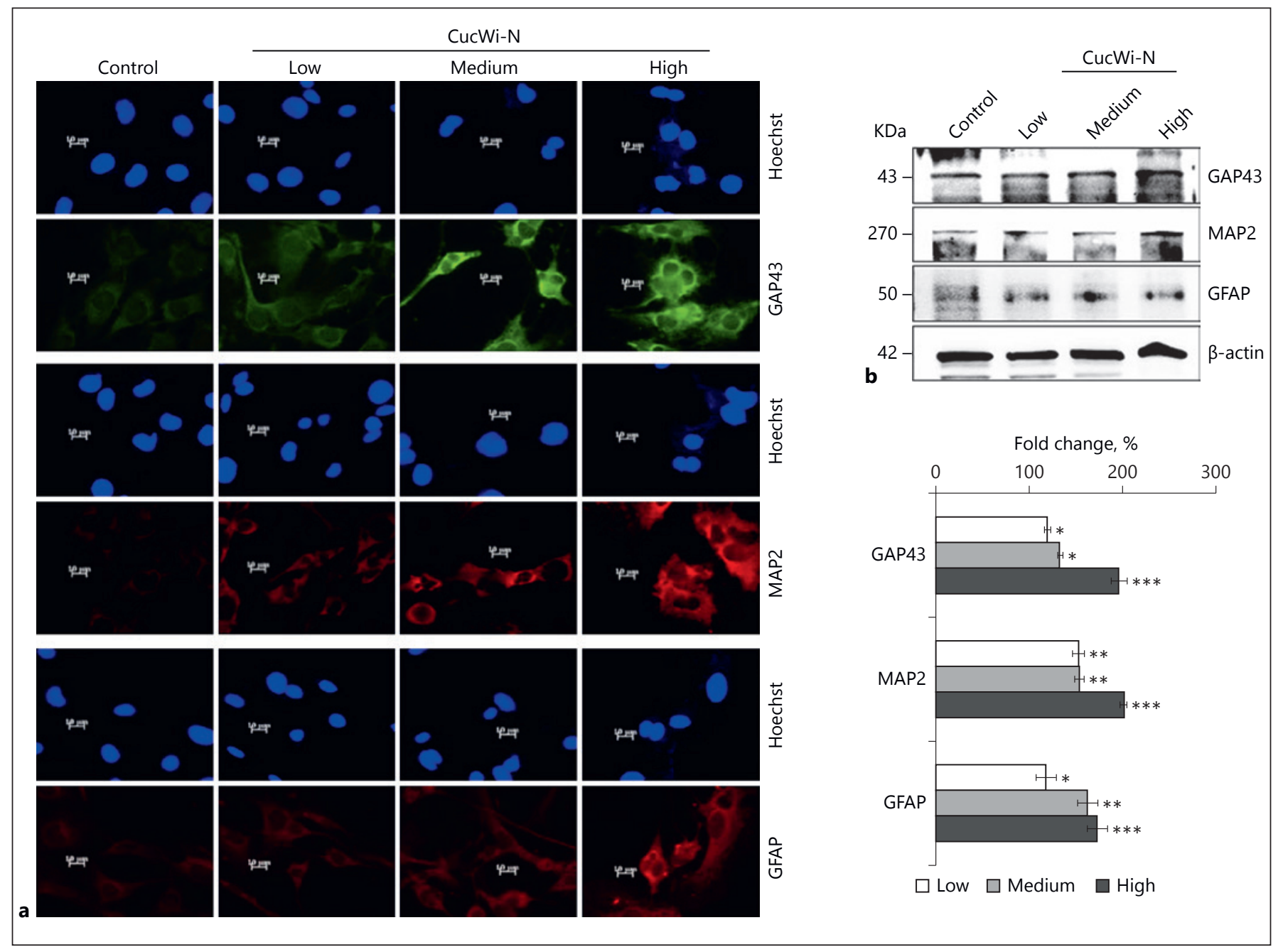

Fig. 4. Effect of CucWi-N on markers for glial differentiation. a Immunostained images of $\mathrm{CucWi}-\mathrm{N}$ treated $\mathrm{C} 6$ cells demonstrating a dose-dependent increase in glial cell differentiating protein markers GAP43, MAP2, and GFAP; (b), immunoblotting im-

Several studies have suggested anti-inflammatory and anti-stress potential of $\mathrm{Wi}-\mathrm{N}[38,39]$; they have attributed this property to the oxidized 5-7 carbons in its chemistry [40] that translates into weak binding affinity to target proteins [19]. In the normal cells, it delayed the onset of stress-induced senescence [27], and protected brain-derived cells from oxidative and glutamate stresses [22]. Moreover, Wi-N also prevented memory loss in mice model against scopolamine-mediated toxicity [20]. It was shown to enhance both short-term and long-term memories by upregulation of proteins involved in memory [41].

In the present study, we used CucWi-N, a combination of Cuc and Wi-N (1:500 molar ratio) that showed

Anti-Stress and Glial Differentiation Effects of CucWi-N ages of CucWi-N treated C6 cell lysates demonstrating dose-dependent increase in glial cell differentiating protein markers (top) with quantitation (bottom). ${ }^{*} p<0.05,{ }^{* *} p<0.01,{ }^{* * *} p<0.001$.

significant protection against a variety of stresses. Furthermore, it induced remarkable differentiation in glial cells in a dose-dependent manner. We propose that Cuc$\mathrm{Wi}-\mathrm{N}$ is a stress protective and glial differentiation inducing combination with potentials for enhancing the QOL in normal or diseased states. Further functional analyses are warranted for its adoption in either nutraceuticals or pharmaceuticals.

\section{Acknowledgments}

S.G. is a recipient of the Honjo International Foundation Scholarship, Japan. 


\section{Ethical Statement}

This study was carried out in strict accordance with the approval of Human-derived Materials Experiments Committee, Safety and Environment Management Division, National Institute of Advanced Industrial Science and Technology (AIST), Japan (Experimental plan approval \#H2014-516).

\section{Disclosure Statement}

Authors do not have any conflicts of interest to disclose.

\section{Source of Funding}

DBT (India) and AIST (Japan).

\section{Authorship Contribution}

S.G. carried out the experiments and reviewed the manuscript. S.C.K. and R.W. designed, supervised, wrote, and reviewed the manuscript.

\section{References}

1 Dong CM, Wang XL, Wang GM, Zhang WJ, Zhu L, Gao S, et al: A stress-induced cellular aging model with postnatal neural stem cells. Cell Death Dis 2014;5:e1116.

2 Saunders LR, Verdin E: Cell biology. Stress response and aging. Science 2009;323:1021-1022.

3 Kumar A, Rinwa P, Kaur G, Machawal L: Stress: neurobiology, consequences and management. J Pharm Bioallied Sci 2013;5:91-97.

4 Moreno-Smith M, Lutgendorf SK, Sood AK: Impact of stress on cancer metastasis. Future Oncol 2010;6:1863-1881.

5 Prasad S, Gupta SC, Pandey MK, Tyagi AK, Deb L: Oxidative stress and cancer: advances and challenges. Oxid Med Cell Longev 2016; 2016:5010423.

6 You W, Henneberg M: Cancer incidence increasing globally: the role of relaxed natural selection. Evol Appl 2018;11:140-152.

7 Hudu SA, Alshrari AS, Syahida A, Sekawi Z: Cell culture, technology: enhancing the culture of diagnosing human diseases. J Clin Diagn Res 2016;10:DE01-DE05.

8 Li L, Gao R, Yu Y, Kaul Z, Wang J, Kalra RS, et al: Tumor suppressor activity of miR-451: identification of CARF as a new target. Sci Rep 2018;8:375.

9 Birben E, Sahiner UM, Sackesen C, Erzurum S, Kalayci O: Oxidative stress and antioxidant defense. World Allergy Organ J 2012;5:9-19.

10 Chen W, Jia Z, Pan MH, Anandh Babu PV: Natural products for the prevention of oxidative stress-related diseases: mechanisms and strategies. Oxid Med Cell Longev 2016;2016: 4628502.

11 Zhang L, Li J, Zong L, Chen X, Chen K, Jiang $Z$, et al: Reactive oxygen species and targeted therapy for pancreatic cancer. Oxid Med Cell Longev 2016;2016:1616781.

12 Carvalho AN, Firuzi O, Gama MJ, Horssen JV, Saso L: Oxidative stress and antioxidants in neurological diseases: Is there still hope? Curr Drug Targets 2017;18:705-718.

13 Uttara B, Singh AV, Zamboni P, Mahajan RT: Oxidative stress and neurodegenerative diseases: a review of upstream and downstream antioxidant therapeutic options. Curr Neuropharmacol 2009;7:65-74.
14 Groenendijk FH and Bernards R: Drug resistance to targeted therapies: deja vu all over again. Mol Oncol 2014;8:1067-1083.

15 Hoelder S, Clarke PA, Workman P: Discovery of small molecule cancer drugs: successes, challenges and opportunities. Mol Oncol 2012;6: 155-176.

16 Rajesh E, Sankari LS, Malathi L, Krupaa JR: Naturally occurring products in cancer therapy. J Pharm Bioallied Sci 2015; 7(suppl 1):S181S183.

17 Wang H, Khor TO, Shu L, Su ZY, Fuentes F, Lee JH, et al: Plants vs. cancer: a review on natural phytochemicals in preventing and treating cancers and their druggability. Anticancer Agents Med Chem 2012;12:1281-1305.

18 Gao R, Shah N, Lee JS, Katiyar SP, Li L, Oh E, et al: Withanone-rich combination of Ashwagandha withanolides restricts metastasis and angiogenesis through hnRNP-K. Mol Cancer Ther 2014;13:2930-2940.

19 Grover A, Priyandoko D, Gao R, Shandilya A, Widodo N, Bisaria VS, et al: Withanone binds to mortalin and abrogates mortalin-p53 complex: computational and experimental evidence. Int JBiochem Cell Biol 2012;44:496-504.

20 Konar A, Shah N, Singh R, Saxena N, Kaul SC, Wadhwa R, et al: Protective role of Ashwagandha leaf extract and its component withanone on scopolamine-induced changes in the brain and brain-derived cells. PLoS One 2011; 6:e27265.

21 Shah N, Kataria H, Kaul SC, Ishii T, Kaur G, Wadhwa R: Effect of the alcoholic extract of Ashwagandha leaves and its components on proliferation, migration, and differentiation of glioblastoma cells: combinational approach for enhanced differentiation. Cancer Sci 2009;100:1740-1747.

22 Shah N, Singh R, Sarangi U, Saxena N, Chaudhary A, Kaur G, et al: Combinations of Ashwagandha leaf extracts protect brain-derived cells against oxidative stress and induce differentiation. PLoS One 2015;10:e120554.

23 Vaishnavi K, Saxena N, Shah N, Singh R, Manjunath K, Uthayakumar M, et al: Differential activities of the two closely related withanolides, Withaferin A and Withanone: bio- informatics and experimental evidences. PLoS One 2012;7:e44419.

24 Widodo N, Takagi Y, Shrestha BG, Ishii T, Kaul SC, Wadhwa R: Selective killing of cancer cells by leaf extract of Ashwagandha: components, activity and pathway analyses. Cancer Lett 2008;262:37-47.

25 Yu Y, Katiyar SP, Sundar D, Kaul Z, Miyako E, Zhang Z, et al: Withaferin-A kills cancer cells with and without telomerase: chemical, computational and experimental evidences. Cell Death Dis 2017;8:e2755.

26 Priyandoko D, Ishii T, Kaul SC, Wadhwa R: Ashwagandha leaf derived withanone protects normal human cells against the toxicity of methoxyacetic acid, a major industrial metabolite. PLoS One 2011;6:e19552.

27 Widodo N, Shah N, Priyandoko D, Ishii T, Kaul SC, Wadhwa R: Deceleration of senescence in normal human fibroblasts by withanone extracted from ashwagandha leaves. J Gerontol A Biol Sci Med Sci 2009;64:10311038.

28 Chaudhary A, Kalra RS, Huang C, Prakash J, Kaul SC, Wadhwa R: 2,3-Dihydro-3beta-methoxy Withaferin-A protects normal cells against stress: molecular evidence of its potent cytoprotective activity. J Nat Prod 2017;80: 2756-2760.

29 Li K, Yu Y, Sun S, Liu Y, Garg S, Kaul SC, et al: Functional characterisation of anticancer activity in the aqueous extract of Helicteres angustifolia L. Roots. PLoS One 2016;11: e0152017.

30 Garg S, Kaul SC, Wadhwa R: Cucurbitacin B and cancer intervention: chemistry, biology and mechanisms (Review). Int J Oncol 2018; 52:19-37.

31 Wadhwa R, Kaul SC, Ikawa Y, Sugimoto Y: Identification of a novel member of mouse hsp70 family. Its association with cellular mortal phenotype. J Biol Chem 1993;268: 6615-6621.

32 Kroschinsky F, Stolzel F, von Bonin S, Beutel G, Kochanek M, Kiehl M, et al: New drugs, new toxicities: severe side effects of modern targeted and immunotherapy of cancer and their management. Crit Care 2017;21:89. 
33 Goodenberger ML, Jenkins RB: Genetics of adult glioma. Cancer Genet 2012;205:613-621.

34 Jessen KR: Glial cells. Int J Biochem Cell Biol 2004;36:1861-1867.

35 Yin D, Wakimoto N, Xing H, Lu D, Huynh T, Wang X, et al: Cucurbitacin B markedly inhibits growth and rapidly affects the cytoskeleton in glioblastoma multiforme. Int J Cancer 2008; 123:1364-1375.

36 Touihri-Barakati I, Kallech-Ziri O, Ayadi W, Kovacic H, Hanchi B, Hosni K, et al: Cucurbitacin $B$ purified from Ecballium elaterium (L.) A. Rich from Tunisia inhibits $\alpha 5 \beta 1$ integrin-mediated adhesion, migration, proliferation of human glioblastoma cell line and angiogenesis. Eur J Pharmacol 2017;797:153-161.
37 Clericuzio M, Mella M, Vita-Finzi P, Zema M, Vidari G: Cucurbitane triterpenoids from Leucopaxillus gentianeus. J Nat Prod 2004;67: 1823-1828

38 Pandey A, Bani S, Dutt P, Kumar Satti N, Avtar Suri K, Nabi Qazi G: Multifunctional neuroprotective effect of Withanone, a compound from Withania somnifera roots in alleviating cognitive dysfunction. Cytokine 2018;102:211-221.
39 Purushotham PM, Kim JM, Jo EK, Senthil K: Withanolides against TLR4-activated innate inflammatory signalling pathways: a comparative computational and experimental study. Phytother Res 2017;31:152163.

40 Joshi P, Misra L, Siddique AA, Srivastava M, Kumar S, Darokar MP: Epoxide group relationship with cytotoxicity in withanolide derivatives from Withania somnifera. Steroids 2014;79:19-27.

41 Gautam A, Wadhwa R, Thakur MK: Involvement of hippocampal Arc in amnesia and its recovery by alcoholic extract of Ashwagandha leaves. Neurobiol Learn Mem 2013;106:177184. 